\title{
Les rebuts captivants
}

Un nouvel ordre bouffon au cœur de la collectivité

Community's Trash, Ritual Clown's Treasure: the Transformation of Cast-offs in the Hands of the Ritual Clown

\section{Laure Carbonnel}

\section{OpenEdition Journals}

Édition électronique

URL : https://journals.openedition.org/tc/8122

DOI : $10.4000 /$ tc. 8122

ISSN : 1952-420X

Éditeur

Éditions de l'EHESS

\section{Édition imprimée}

Date de publication : 31 octobre 2016

Pagination : 370-385

ISBN : 9782713225291

ISSN : 0248-6016

Référence électronique

Laure Carbonnel, «Les rebuts captivants », Techniques \& Culture [En ligne], 65-66 | 2016, mis en ligne le 31 octobre 2018, consulté le 29 septembre 2022. URL : http://journals.openedition.org/tc/8122 ; DOI : https://doi.org/10.4000/tc.8122 


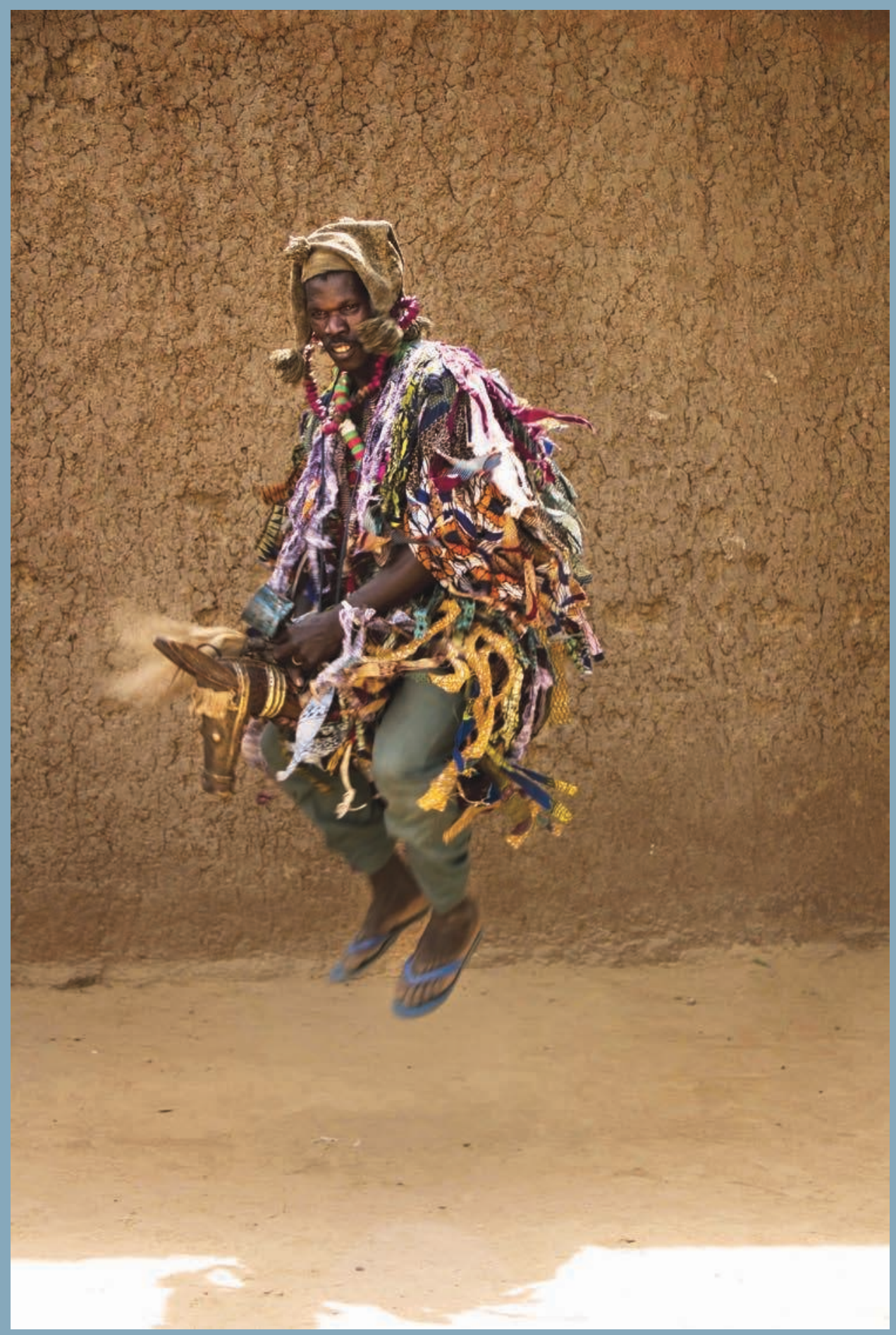




\section{Les rebuts captivants}

\section{Un nouvel ordre bouffon au cœur de la collectivité}

Au Mali ${ }^{1}$, des bouffons rituels (kóródugaw ${ }^{2}$ en bambara) colorent les rues et les cérémonies de leurs parures composites et de leurs comportements simultanément joyeux et déconcertants. Les principaux signes qui permettent de reconnaître ces bouffons, parures, danses et prénoms, sont qualifiés en bambara et par eux-mêmes de kolon, qui signifie usagé, vieux, dans le sens d'inutile ${ }^{3}$. Leurs parures sont confectionnées à partir de vieux habits usés et troués auxquels s'ajoute une multitude de fragments collectés dans leur environnement. Les prénoms qu'ils attribuent aux nouveau-nés intégrés dans la bouffonnerie sont dits sans valeur: "vieux panier» (Sekin kolon), vieux déchet (Nyamakolon ${ }^{4}$ ), ou encore «sans honte» (Tietemalo ou Malobali) 5 .Gloutons, les bouffons mélangent tout sans discernement en laissant des traces autour de la bouche. Enfin, l'un de leurs rôles est de protéger femmes et enfants socialement marginalisés. Autrement dit, le principal attribut du bouffon rituel est d'exposer tout ce qui ne trouve plus sa place dans la collectivité.

La valeur donnée aux restes et à leur usage varie selon les sociétés et selon le type de déchets concernés. Au Mali, restes et objets usagés sont soumis à une gestion structurée. Les déchets humains sont rigoureusement contrôlés pour éviter qu'ils ne soient récupérés par une personne mal intentionnée, car, comme les noms, les déchets restent attachés à la personne et peuvent être employés pour influencer ou rendre malade son propriétaire (Dumestre \& Seydou 2007). À l'inverse, objets et matières confectionnés - récipients ferrailles, habits, etc. entrent dans des circuits de réparation, de récupération, de transformation et de réemploi. Ce processus donne une seconde vie à toute chose aliénable ou inaliénable devenue inutile, parce que cassée, trouée, séparée du tout dont elle faisait partie, débarrassée de son contenu ou détachée d'un propriétaire à même d'en faire usage.

Parallèlement à cette circulation occulte des déchets humains et à cette circulation publique des objets de seconde main, les bouffons rituels ouvrent une troisième voie. Nous 
allons voir qu'ils recomposent certains matériaux usagés pour forger un «soi » bouffon, puis qu'ils réintègrent les rebuts ${ }^{6}$ au cœur de la société en s'invitant dans les maisons et les cérémonies. Ces deux processus bouffons de recomposition et d'exposition ne redonnent pas seulement une valeur à ces matériaux, ils ouvrent de nouvelles relations de causalités entre objets, personnes et fonctions.

\section{La bouffonnerie, une esthétique de l'inutile}

\section{Des restes alimentaires ingurgités}

Exubérance, gloutonnerie, exhibition de la sexualité et de la scatologie, les manières bouffonnes ont un côté rabelaisien (Colleyn 2010). D’un type particulier néanmoins, car si les bouffons mangent beaucoup, c'est avant tout les restes qui les intéressent.

Tout processus de transformation produit déchets et résidus, en premier lieu la cuisine. Lorsque le plat national, appelé to, est cuit, la nourriture est transvasée de la marmite au récipient de service. Pour éviter que cela n’accroche trop, la cuillère est humectée dans une calebasse d'eau, avant de plonger dans la marmite encore chaude. À chaque geste, un peu de to resté sur la cuillère vient se mélanger à l'eau appelée toboji «l'eau pour sortir le to ». Cette eau, les bouffons en représentation n'hésitent pas à la boire alors qu'elle est ordinairement jetée par la cuisinière ou réutilisée pour laver la marmite. Toboji est aussi l'un des prénoms sans valeur attribués aux enfants dont les aînés sont tous décédés en bas âge, des enfants pris en charge par les bouffons comme nous le verrons plus loin.

La qualité de reste n'est pas un état en soi, elle est aussi donnée par la manière de consommer les aliments. Gloutons, les bouffons passent rarement à côté d'un plat, d'une marmite, ou même d'un mortier sans avaler goulûment quelques poignées de nourriture dont ils laissent une trace autour de la bouche. Ils mangent et mélangent tout sans discernement, du plat cuisiné à la farine fraîchement pilée. Se faisant, ils transforment la valeur accordée à l'alimentation. Les bouffons n'étant jamais rassasiés, les aliments ordinaires perdent leur fonction principale, celle de combler la faim. Lorsqu'ils se goinfrent, qu'ils refusent de partager, ils défient toutes les règles de commensalité, un opérateur social pourtant fondamental (Bloch 2010). L'on pourrait dire que les manières bouffonnes tendent à donner aux aliments le statut de reste à la marge de la sociabilité.

Ces ponctions gloutonnes sont considérées comme positives, car elles annoncent ou favorisent la prospérité agricole. Une dimension propitiatoire que l'on retrouve en pays dogon lors de la consommation de bière cérémonielle produite à partir des surplus de céréales: les restes alimentaires ont un rôle central dans les rituels visant à assurer la continuité « du procès agricole et du cycle du grain» (Jolly 2004: 307). Cependant l'usage que les bouffons font des restes déborde cette seule fonction nourricière. Ils sont aussi réputés pour émettre des flatulences ou pour parler de scatologie. Même si l'on reste ici dans un procès de transformation, l'exposition de la scatologie 
n'a pas de valeur propitiatoire, en revanche elle participe d'une recomposition bouffonne que nous allons aborder par un autre biais, celui de la parure.

\section{Tissus usés et objets cassés exposés dans les parures}

Une troisième manière de manipuler les restes, dans leurs parures, nous permettra de mieux saisir ce qui est en jeu. Ce n'est pas la tenue qui fait le bouffon, car au Mali il s'agit d'un statut transmis par filiation, la liberté étant laissée à chacun de s'engager dans les représentations bouffonnes, avec ou sans habit spécifique. En revanche, les membres les plus actifs sont sévèrement critiqués s'ils ne portent pas une tenue bouffonne lorsqu'ils se donnent en représentation. La raison n'est pas seulement identitaire, nous allons voir que c'est aussi une manière de se positionner dans la collectivité et d'agir sur elle.

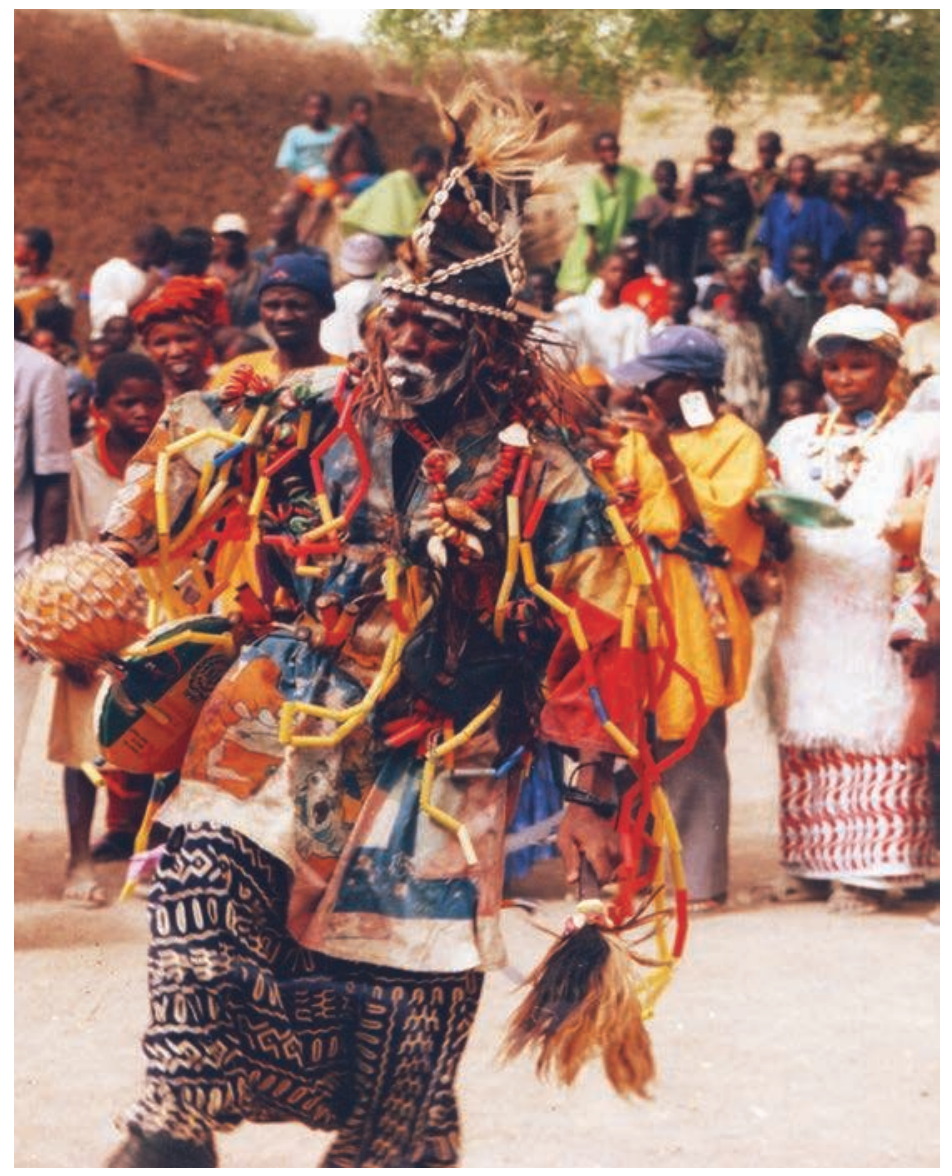

Certaines parures bouffonnes ont une forme très spécifique. L’une, réservée aux hommes, est une tunique recouverte de maintes lanières découpées dans des tissus de coton ou constituée d'une enfilade de restes de cartouches, recouvrant le corps à l'image des masques rituels et virevoltant à chaque pas de danse.

Dans les années soixante, une photo prise par D. Zahan (1960) montre une tenue similaire faite de tiges de mil, récupérées après le battage. Un autre type de tenue caractéristique des bouffons est un pantalon et une tunique cousus dans des sacs de céréales.

La majorité des bouffons se contentent néanmoins de tenues cousues à partir de pagnes communs, qui se distinguent essentiellement
Tunique en patchwork recouverte d'enfilades de restes de cartouches virevoltants à chaque pas de danse. Le président de l'«association de korèdugaw de Ségou » à Farako en 2006. 


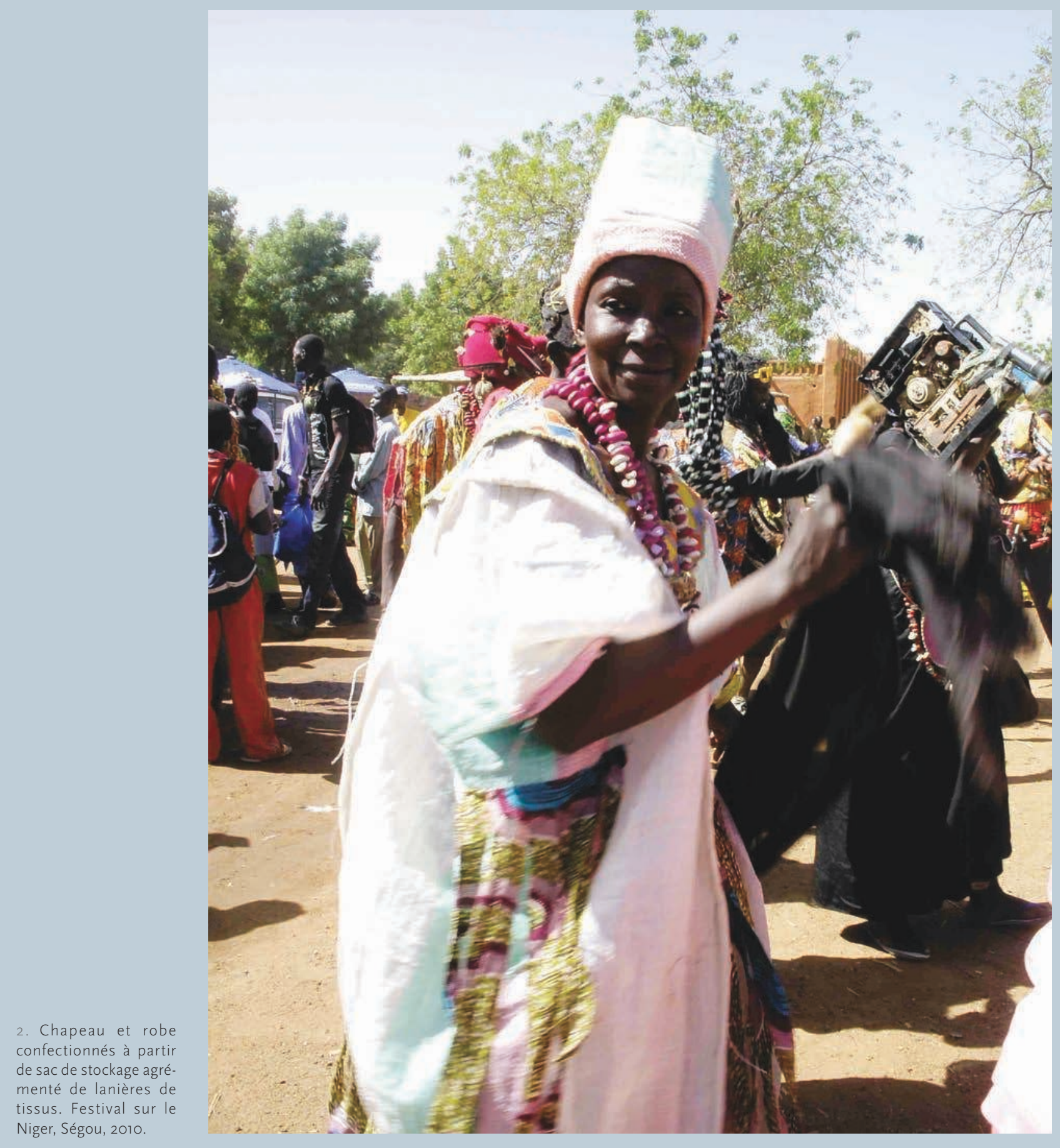


par leur caractère usagé. Les femmes bouffonnes portent fréquemment un pantalon bouffant au lieu d'une jupe, et souvent une tunique en patchwork (des morceaux de vieux tissus cousus ensemble). Les parures les plus simples des hommes sont pour beaucoup des tenues de chasseurs (qui forment au Mali un groupe influent), mais là encore particulièrement usées. D’une manière générale, hommes et femmes portent des tenues ordinaires montrant une activité, comme une tenue militaire ou une tenue de chasseurs, ou ses marques comme le t-shirt maculé d'un réparateur de moto.

Les tissus neufs ne sont pas proscrits, mais dans ce cas, les bouffons rituels ajoutent des lanières de tissus découpées dans de vieux habits, ou d'autres accessoires comme des vieilles bouteilles en plastique, qui viennent en quelque sorte contaminer l'ensemble du vêtement.

Laccumulation, d'accessoires, de vêtements ou de vieux objets dépareillés, est aussi un procédé central des tenues bouffonnes. Sur la tête, hommes et femmes portent de vieilles perruques mates (les perruques neuves des femmes sont brillantes), courtes ou longues, des chapeaux, des bonnets colorés ou des oreilles d'âne. Sur le visage, les bouffons ajoutent de larges lunettes en calebasse ou des vieilles lunettes cassées. À la taille, ils portent des bouteilles en plastique, des ceintures faites avec des capsules, etc. Sur le torse, retombent des bouts de filets de pêche ou tout autre objet du quotidien comme une embrasse de rideau. Les bouffons accessoirisent jusqu'à l'élément qui est le plus attaché à leur identité, leur collier de fèves rouges auquel ils ajoutent des perles en plastique, des cartouches, des coquillages, des capsules, des arachides parfois des sachets de plantes médicinales.

Ainsi, par emprunts, superpositions, recompositions de vieux habits troués ou rapiécés, les bouffons s'habillent de matériaux qui tendent à être mis de côté, qui ont perdu leur utilité, ou qu'ils détachent de leurs fonctions initiales. Ces matières et objets sont récupérés à « un moment de la vie d'un objet» (Dupré2007: 29). Il s'agit d'objets dont il est difficile de trouver une autre fonction, comme un tissu trop usé ou un récipient trop cassé pour pouvoir être réparé, d'objets détournés de leur fonction initiale, comme un jouet porté à la taille, ou des matériaux détournés de leur usage ordinaire, les habits empruntés au sexe opposé notamment. S'ils ne sont ni jetés, ni réparés, quel autre sort leur est-il réservé?

\section{Des parures composites et composées}

Les rebuts sont parfois stylisés, comme les cartouches de fusil. Mais ils ne sont pas totalement transformés, dans le sens où l'on reconnaît toujours facilement l'objet initial. Les artéfacts fabriqués par les bouffons sont de confection intentionnellement grossière. Par exemple, si l'on reconnaît une caméra (au fond à droite), la volonté est moins d'imiter que de suggérer, puisque l'on voit clairement qu'il s'agit d'une unité centrale cassée et de deux lampes torches attachées avec des morceaux de tissus et de caoutchouc.

Les vêtements accentuent cet effet composite, car aucune figure ne se détache réellement de l'accumulation d'éléments. Faisant corps avec leurs porteurs, les vêtements ne se laissent 
3. Femme kóróduga munie d'un artéfact composite qui préserve l'identité de chacun de ses éléments (lampe torche, bouteille plastique, coude), porté ici comme un fusil face à un soldat assis. pas aisément décomposer, il ne s'agit pas d'objets figés tel un tableau sur lequel l'œil peut se promener à son aise. D'autant que même si l'identité de chaque objet est maintenue, la fonction initiale est détournée. Les contenants comme les sacs de céréales deviennent des vêtements. Les lunettes de soleil, pourtant bien portées sur le nez, n'atteignent jamais leur fonction ultime: les verres sont soit inexistants, soit cassés. Ainsi, les parures bouffonnes forment un ensemble flou d'éléments disparates non hiérarchisés, plus qu'elles exposent des symboles affichés de statuts pris et montrés.
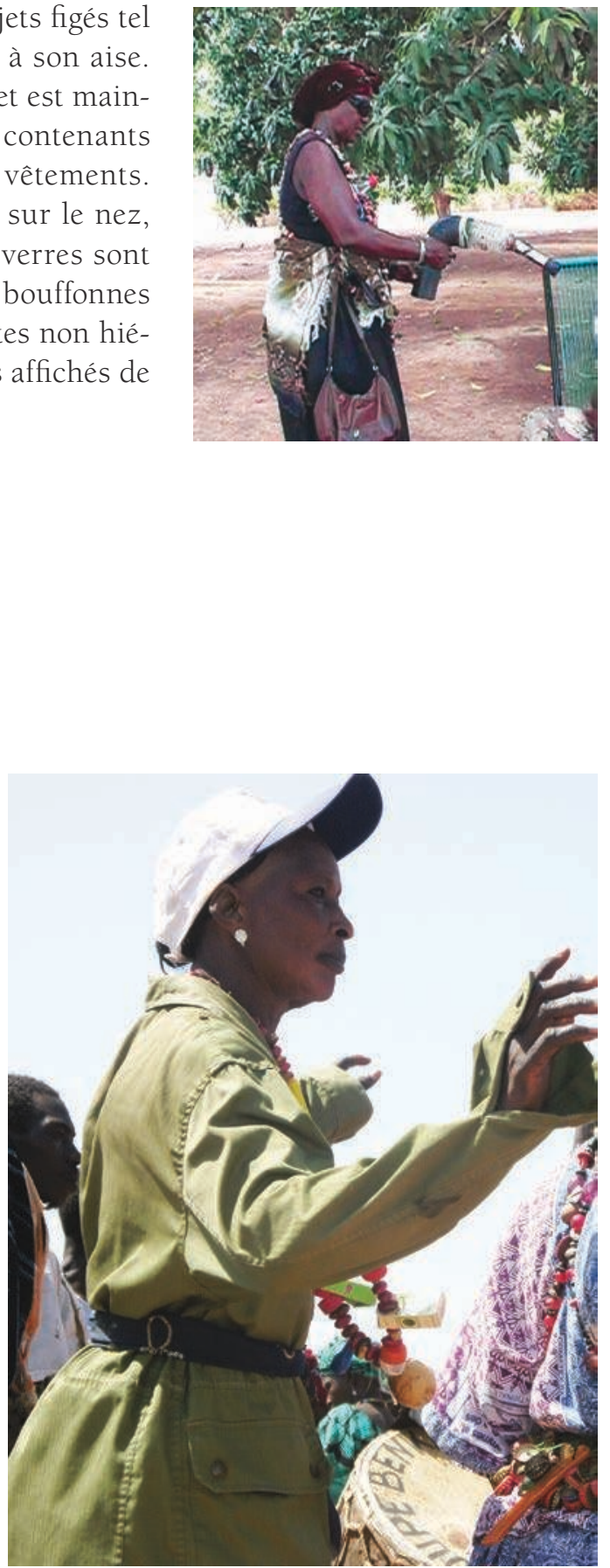

4. Tunique ordinaire cousue dans un tissus neuf. A la ceinture, de vieilles bouteilles plastiques écrasées et poussiéreuses. Fête kóróduga à l'occasion du cinquantenaire de l'indépendance, Ségou, 2010.

5. Chemise de militaire empruntée par une femme kóróduga et collier agrémenté de paquets de thé. Fête d'inauguration des travaux routiers, Ségou, 2010.

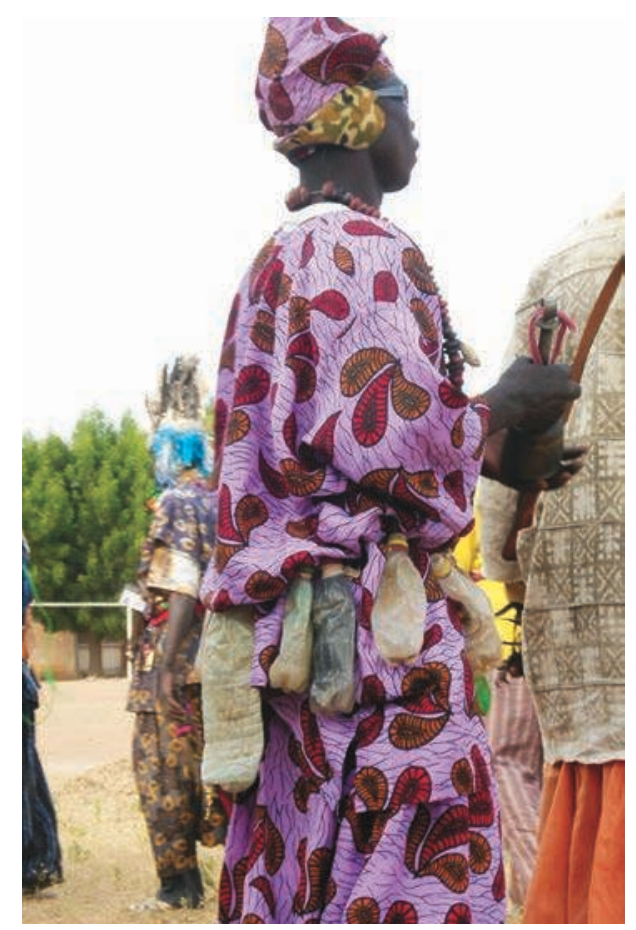


Cette composition particulière fait que les bouffons ne se confondent jamais avec ce que leurs accessoires représentent. Par exemple, le porteur d'oreilles et de pattes d'âne ne fait pas nécessairement l'âne dans ces mouvements, plus encore, n'importe quel bouffon avec ou sans parure peut jouer l'animal. Au contraire, en confectionnant des parures à partir de restes, les bouffons dissocient le rapport entre l'activité et l'acteur. Ainsi par exemple, une femme affublée du t-shirt maculé d'un mécanicien n'est pas identifiée comme un garagiste ou un réparateur de moto. Autrement dit, ce sont les traces d'activités qui représentent au mieux l'identité bouffonne, plus que les bouffons ne représentent une activité donnée. Et ce sont les mouvements bouffons, sauts et pas dansés, qui finissent de structurer cette esthétique bouffonne particulière.

\section{Singularités vs similitudes}

Au Mali, une forte valeur identitaire est conférée aux vêtements. Les chasseurs par exemple ont une tenue distinctive adaptée à l'activité cynégétique - des vêtements réalisés à partir de tissus teints selon une technique particulière appelée bogolan, mélange à base de terre et de plante appliqué essentiellement sur un coton épais qui absorbe les humeurs du corps (Duponchel 1999: 223-224). Les chasseurs portent cette tenue également lors des rassemblements festifs. Par effet de similarité, la tenue se détache alors de sa simple dimension fonctionnelle, pour devenir un signe d'appartenance. Cet effet de groupe est fréquemment recherché dans la région de Ségou au Mali. Les membres d'une même association organisent systématiquement l'achat d'un tissu identique pour en faire un vêtement qu'ils porteront au même moment, de manière à afficher une adhésion commune. Il en va de même pour les proches des époux le jour de leur mariage. Autrement dit, l'unité du groupe est très prégnante, elle se manifeste habituellement par des tenues vestimentaires similaires attachées à une activité, à une catégorie, à un événement, à une relation.

Les bouffons ne dérogent pas à cette norme, comme nous l'avons évoqué plus haut, le refus de porter une tenue bouffonne est mal perçu lorsqu'ils se rassemblent pour partir pratiquer la bouffonnerie ensemble.

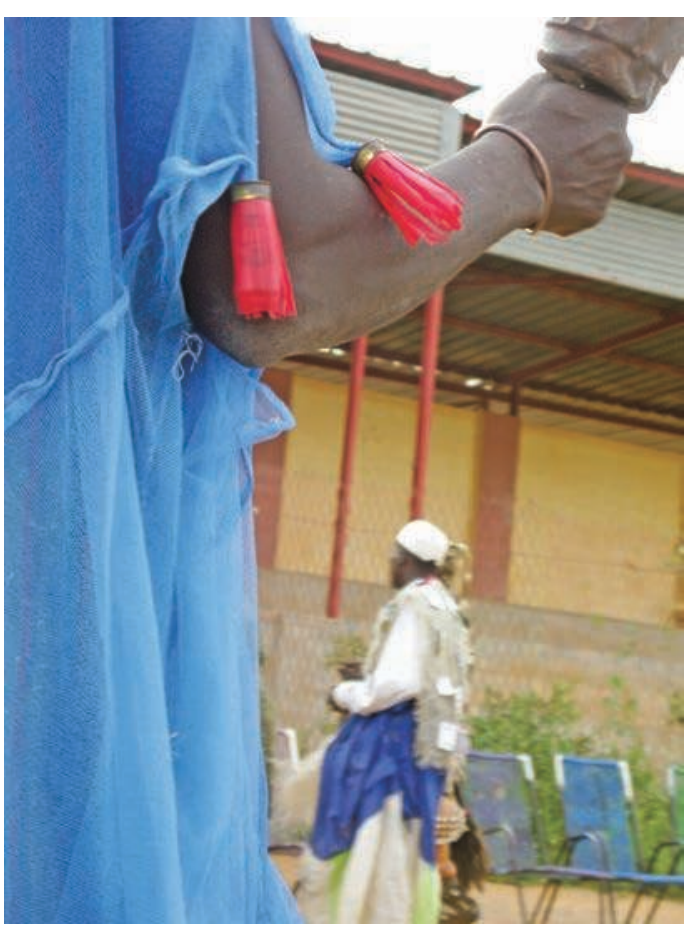

6. Tunique faite à partir d'une moustiquaire déchirée, accessoirisée par des embouts plastiques de cartouches de fusil effilées. A la main, un hochet métallique fabriqué à partir d'une petite bonbonne de gaz vide. Ségou, 2010. 
7. Tenue de jeune adulte commune (short, t-shirt, tunique traditionnelle) sur laquelle sont ajoutés lanières et accessoires attachés à la ceinture (cartouches, boîtes de médicaments, lanières de tissus, embrasure de rideau) virevoltants sur les pas de danses sautés. Ségou, 2010.

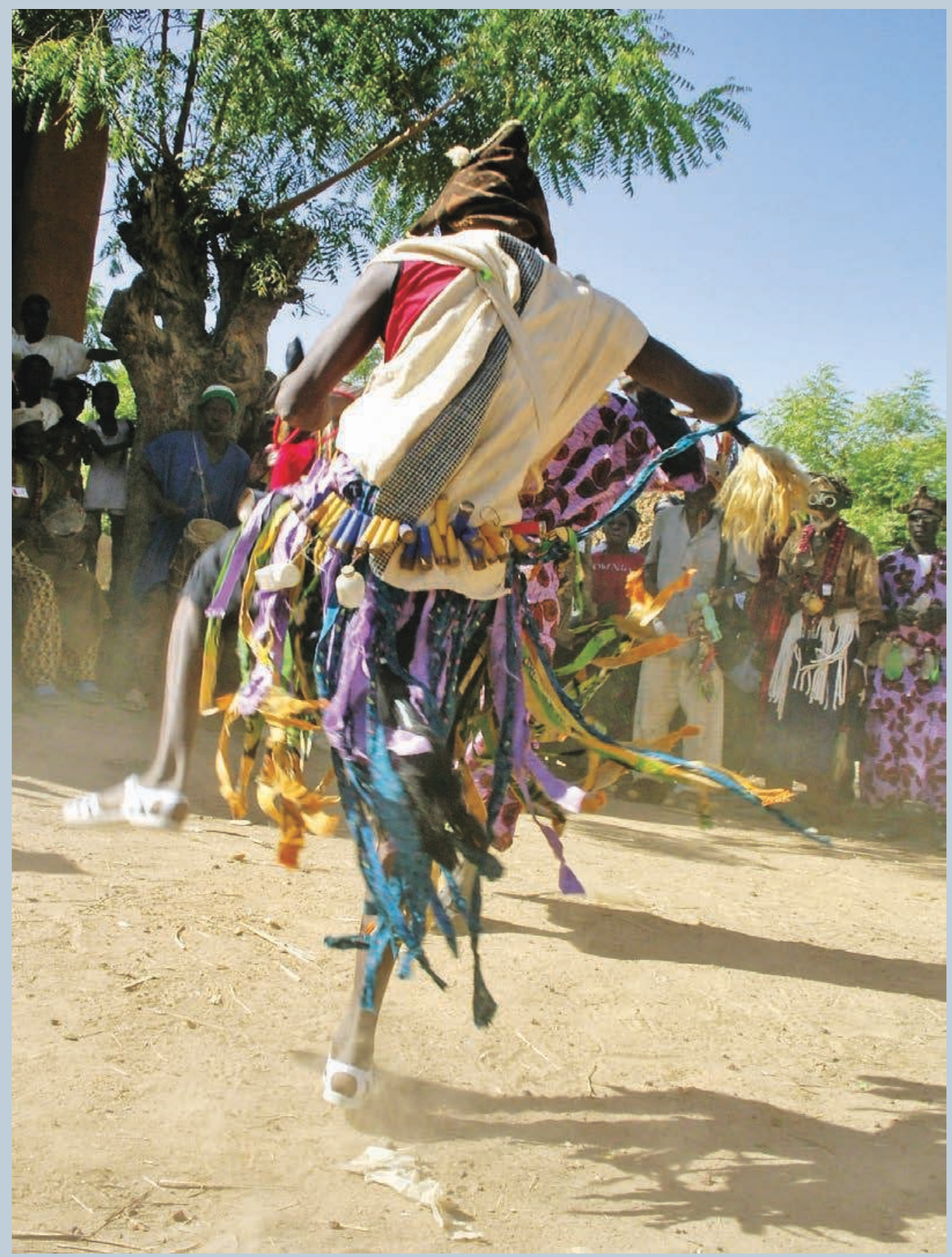


Leur particularité est d'élaborer cette identité commune sur la base de singularités plutôt que par effet de similitude. Comme le remarqua déjà Monteil (1924), le choix et la composition d'une tenue sont laissés à la discrétion de chaque bouffon. Chaque membre aura donc une parure différente, car chacun puise dans son environnement social pour la confectionner. Le bouffon forgeron vient avec ses instruments en fer; celui qui réside au bord du fleuve et côtoie les pêcheurs prendra un filet; une bouffonne cuisinant pour une troupe d'artistes vêtus d'une tunique aux couleurs du drapeau malien, réutilisera cette tunique pour ses propres fêtes bouffonnes.

Les parures sont aussi choisies en fonction de leurs qualités attractives et de leurs potentiels ludiques lesquels nourrissent interactions et jeux bouffons. Elles sont plus ou moins drôles, plus ou moins déroutantes, et les bouffons n'ont de cesse de commenter les vêtements et les accessoires des uns et des autres. Dès qu'une idée plaît, elle est immédiatement imitée. En suscitant des envies, l'accessoire se diffuse et devient une référence esthétique attachée à la bouffonnerie.

En résumé, l'identité bouffonne émerge du côté usé et composite des matériaux utilisés, et de la disjonction entre une tenue et son référent initial. Elle émerge aussi du pouvoir attracteur de certaines parures qui conduit par rétroaction à l'usage des mêmes types d'accessoires. Enfin, les bouffons que j'ai suivis prennent soin de former visuellement un groupe en jouant sur le principe de proximité dans le mouvement musical et dansé des processions en ligne et des danses en cercle.

\section{Des restes réintroduits au cœur de la société}

Ainsi parés et identifiés, les bouffons entrent dans les maisons, dans les marchés et dans les cérémonies, ce qui implique d'assumer une certaine position.

Un jour, alors que nous discutions dans une cour, un chef kóróduga m'interroge: «Je suis invité à la fête d'intronisation d'un chef griot, quelle tenue dois-je porter? » Face à mon regard interrogateur, il poursuit: "Je ne sais pas si je dois porter une tenue de bouffon pour faire une animation ou un beau boubou si je viens en qualité d'invité car je suis un chef d'association. » Il choisira une parure intermédiaire, un pantalon bouffant et une tunique cousue dans un tissu dédié à la fête du Travail. Le choix de cette tenue lui permettra de naviguer parmi les participants dans une modalité relationnelle bouffonne atténuée, c'est-à-dire sans s'imposer visuellement aux autres en tant que bouffon. Le port d'une parure bouffonne montre non seulement l'engagement de l'individu dans la bouffonnerie, mais aussi la place de la bouffonnerie dans l'événement auquel il participe.

\section{Une question de contenance}

La question initiale de mon interlocuteur, «comment dois-je m'habiller?», signifiait dans le même temps «comment dois-je me comporter?» En effet, au-delà du symbole identitaire, la qualité d'un 


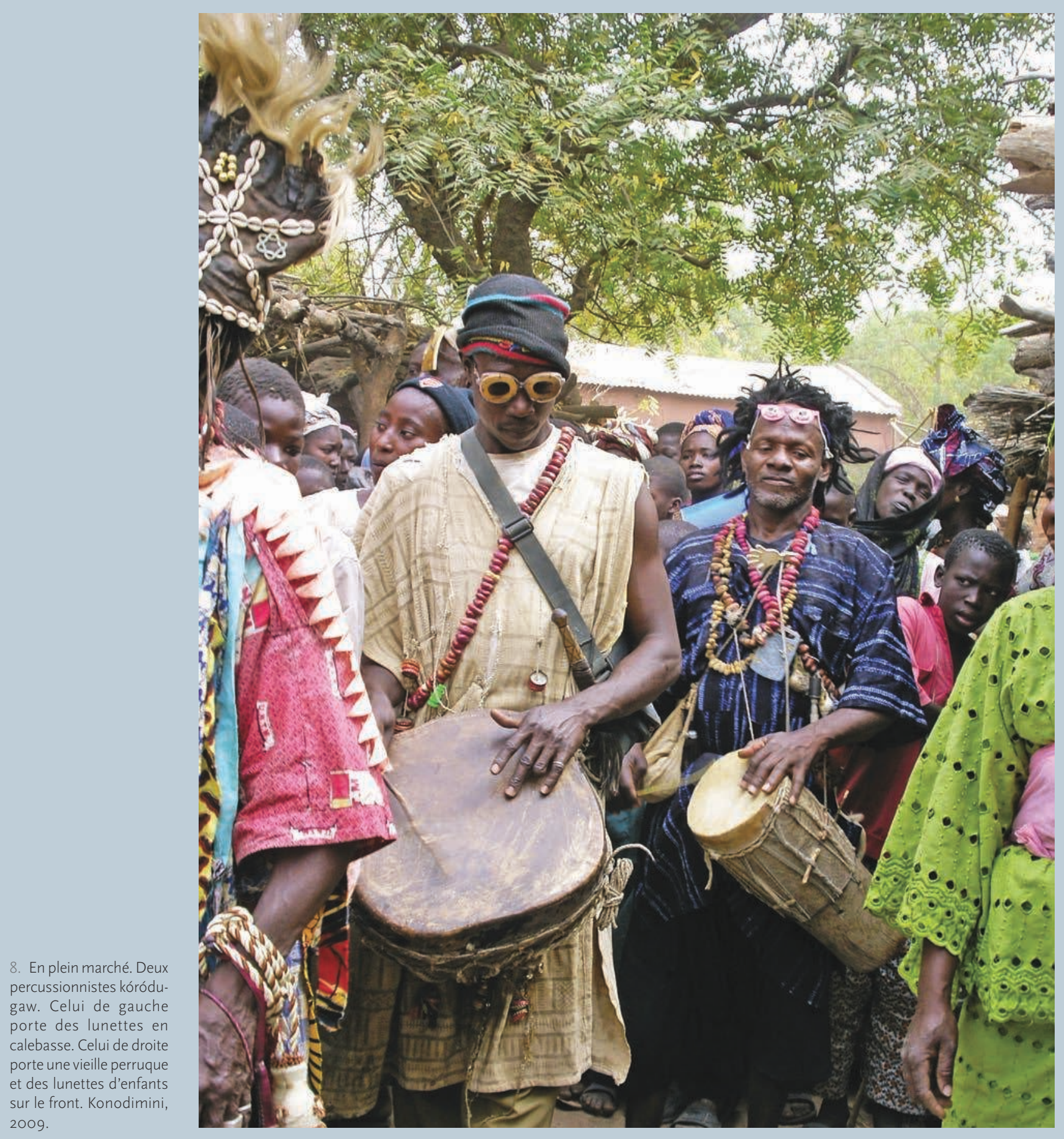




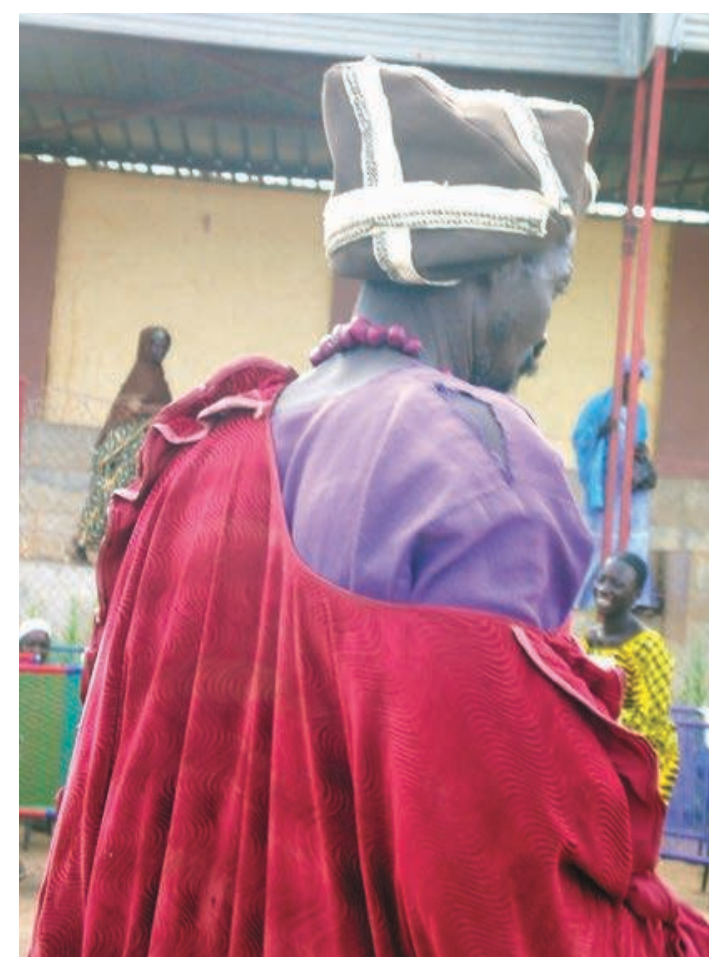

vêtement implique également une contenance que son porteur veut se donner, celle qu'il veut montrer. Au Mali, les femmes mettent par exemple leurs plus vieux habits pour s'occuper des tâches ménagères, en revanche, pour voyager en bus, elles revêtent fréquemment leurs plus beaux habits alors que la chaleur et la poussière rendent le voyage inconfortable et salissant. Autrement dit, le choix de la parure reflète moins l'environnement que l'attitude et le statut que son porteur veut afficher.

La question peut d'ailleurs être inversée: «comment dois-je m'habiller pour me comporter ainsi?». Pantalons bouffants, vieux habits ou matières insalissables (le bogolan en coton épais ne se lave pas, de même que les tenues en sacs de céréales) s'ajustent aux mouvements bouffons, leurs sauts à terres, leurs déambulations. Porter une tenue marquée par l'usure, leur laisse toute liberté dans les activités qu'ils veulent mener.
9. Faire d'un cumul de vieux habits des tenues de cérémonie. Parure d'homme kóróduga (de dos): une tunique trouée bleue surmontée d'une robe usée. Fête à l'occasion du cinquantenaire de l'indépendance, Ségou, 2010.

\section{Exposition de la marge}

Les parures des bouffons ont aussi un impact dans leur rapport aux autres, car ils font de vieux habits des parures de cérémonie, là où les participants achètent au contraire de riches parures neuves pour l'occasion, des vêtements «non déformés par les mouvements propres à celui qui les porte» (Simmel 1998: 83), notamment pour les cérémonies de mariage en milieu urbain.

Le contraste entre les participants richement vêtus et les bouffons vêtus de haillons pourrait apparaître comme l'intrusion de la pauvreté chez les plus riches, comme la critique d'un excès de consommation ou d'ostentation. Pourtant, les bouffons ne s'affichent pas comme des représentants des pauvres ou des critiques des riches, leur absence d'ostentation volontaire n'est pas orientée dans ce cas vers la satire. Au contraire, certains aiment affirmer leur statut de noble, et ils prennent toujours grand soin à confectionner leurs parures. Pour éviter d'apparaître comme des nécessiteux, des quémandeurs, même s'ils suscitent des dons, leurs activités quotidiennes, de planton, de forgeron, de maraîcher, de marchand, etc. sont mises en avant lorsqu'ils se présentent verbalement. Les bouffons sont bien critiques, et satiriques, mais seulement envers ceux qui, par exemple, sont moralisateurs alors que leur conduite est 
immorale ou intéressée, ou bien, autre exemple, envers un juge injuste. Autrement dit, cette critique porte moins sur un état d'être, que sur une discordance entre une position ou un discours et l'action effective de leur cible.

Ce contraste entre parures montre davantage une absence de positionnement envers l'événement et envers les statuts, une manière de s'abstraire de toutes les relations censées influencer les comportements. En effet, d'ordinaire, tout un chacun change de tenue en fonction du milieu social dans lequel il se montre, les parures des griots par exemple sont à l'image de leur notoriété et de leurs relations avec les organisateurs des cérémonies. Au contraire, les bouffons portent les mêmes tenues, et usent des mêmes procédés, quel que soit leur statut (en dehors de celui de bouffon), quelle que soit l'occasion, qu'ils interviennent chez un organisateur riche et influent, ou chez un villageois désargenté, qu'ils se rendent à une grande cérémonie de mariage ou à une petite fête de quartier.

Autrement dit, les bouffons s'imposent en exposant au grand jour tout ce qui est délaissé, mis de côté ou caché, sans leur conférer une nouvelle utilité, sans se laisser socialement dévaloriser, et sans être influencés par l'attitude attendue en public dans une situation donnée. Ils imposent leur propre ordre bouffon, tout en se fondant dans la société par respect d'un certain nombre de conventions.

\section{En miroir des personnes marginalisées, le potentiel bouffon}

Ces personnages présentent ainsi ce que l'on pourrait appeler « une manière d'être qui ne possède rien » pour être en relation avec «ceux qui possèdent pour être ». Cette exposition de l'absence de valeur est un moyen de protection. C'est aussi un «pouvoir faire» qui remodèle et réintègre tout ce qui a été délaissé dans la société, les personnes marginalisées en particulier.

La position assumée par les bouffons en représentation, avec ses conduites, ses mimiques, ses objets cassés et ses prénoms sans valeurs exposés, acquiert une efficacité, lorsqu'elle est orientée vers certaines personnes marginalisées ${ }^{7}$. Au Mali, comme dans d'autres sociétés, une affliction touche nombre de femmes mariées: l'infertilité ou la mort des nourrissons. Or, bien souvent, les femmes bambara au Mali comme les femmes diola au Sénégal n'accèdent «à la plénitude de leur statut social qu'au travers de la procréation ». (Journet 1981: 101). Un enfant procure à sa mère une place dans la maison de son mari, dans les associations féminines; il lui donne pour ainsi dire accès à la vie publique de son quartier (Journet 1981 : 102). Les femmes mariées sans enfants sont insidieusement mises au ban, sans jamais être exclues totalement puisqu'elles restent chez leur mari: à ma connaissance l'absence de progéniture ne peut justifier à elle seule un divorce. Pour traiter cette affliction, la mère peut s'adresser à différents officiants qui agiront en lui fournissant des médecines et en invoquant des entités suprahumaines; aux forgerons par exemple, qui fabriquent et réparent des objets, autant qu'ils assurent la cohésion sociale par leur rôle de médiation (Malé 2007: 43). Il est dit qu’une mère fait appel aux bouffons kóródugaw, en dernier recours. Si elle est en bonne santé et qu'elle vient d'accoucher, elle fait mine de donner le nouveau-né aux 
bouffons pour lui éviter le sort de ses précédents nourrissons décédés en bas âge. L’enfant reste dans sa famille, mais les bouffons lui attribuent un nom sans valeur - «vieux panier », « ordure », etc. - lui fournissent des médecines et l'intègrent dans un nouveau réseau social.

Les bouffons rituels traitent aussi publiquement l'épreuve sociale subie par leurs patients, mère et enfant. Ces derniers sont dans une position similaire à celle mise en scène par les bouffons. Leur situation présente en effet une disjonction entre statut et rôle, puisqu'une femme mariée est reconnue comme telle à partir du moment où elle devient mère, et qu'un enfant est censé survivre aux membres de la génération aînée. La dissonance est grande pour la mère et les enfants à l'instar des personnes qui sont la source de leur marginalisation, car s'ils ne peuvent pas être totalement intégrés dans la collectivité, il est aussi hors de question qu'ils soient complètement rejetés. Les bouffons procèdent avec les femmes et les enfants de la même manière qu'ils exposent les restes de la société, les exhibant publiquement dans leur cercle de danse, et en les intégrant à eux (les femmes et les enfants «traités» deviennent bouffons). Ce faisant, les bouffons montrent que ces personnes marginalisées sont maintenant sous leur protection. Ils rappellent la valeur de ces personnes marginalisées et le rôle de chacun dans l'exclusion ou l'inclusion. Ils les replacent au cœur de la société avec leur affliction, de manière à dénoncer ses causes au lieu de marginaliser la cible.

Pour conclure, les interventions des bouffons fondées sur l'appropriation et l'exposition de tout ce qui est devenu inutile, se placent dans cet interstice décrit par J. Gouhier (1999: 80): «entre l'être et le non-être existe le peut-être». Ils donnent une nouvelle visibilité à ce qui peut paraître hors fonction, sans pour autant attribuer à ces matériaux une nouvelle finalité outre celle d'être exposée. Ils s'approprient aussi les restes de nourriture et bénissent les céréales qui leur sont données pour assurer la prospérité alimentaire des maisonnées. Ainsi, l'être, le non-être et le peut-être ne sont pas des états de fait, les bouffons soulignent que tout cela ne tient finalement qu'à ce qui en est fait. Leur esthétique de l'inutile, leurs expositions des traces d'activités, les résidus qu'ils recomposent à volonté et le traitement en miroir des personnes marginalisées font lien et valorisent l'existence durable des choses au-delà d'une finalité, toujours fragile et éphémère.

\section{Notes}

1. Cet article s'appuie sur un terrain de recherche qui s'est déroulé dans la région de Ségou entre 2004 et 2010, au sein de l'association de korèdugaw, nalomaw et wolosow de Ségou, dans le cadre d'une thèse en anthropologie soutenue en 2015. Je remercie l'ensemble des membres de l'association, dont son président et sa famille, ainsi que les relecteurs anonymes de cette contribution.

2. Nous avons adopté la transcription en phonétique internationale ó est un o ouvert. Une transcription courante est «korèduga», qui est celle choisie par l'association que j'ai suivie sur le papier. Ma transcription suit la prononciation la plus fréquente à Ségou, les deux sont équivalentes pour mes interlocuteurs. Pour des études antérieures sur ces personnages au Mali, voir notamment Monteil 1934, Zahan 1960, Colleyn 1988 et 2010.

3. Et suivant le dictionnaire «paresseux, faible; usagé; vieux, en mauvais état; mauvais; violent; qui ne vaut rien; nu, vide» (Dumestre 2011: 533). 
4. nyama, est aussi le nom de la force vitale, «parfois dangereuse, parfois bénéfique, contenue dans les êtres, les objets sacrés, le ciel, la terre, etc. » (Colleyn 1975: 118).

5. Les femmes ont d'une manière générale recours à ce «camouflage sémantique» (Zonabend 1999: 94) lorsque plusieurs de leurs enfants décèdent en basâge. Certains d'entre eux sont confiés aux kóródugaw, un de leurs rôles principaux étant de protéger femmes et enfants.
6. La notion de rebut est considérée ici dans le sens de chose délaissée, sans connoter nécessairement une idée de souillure. J'emploierai également la notion de reste, qui se situe entre le « déchet » du processus technologique et le «rien» qui est l'inutile, le ridicule, le grotesque suivant la terminologie donnée par Beaune (1999: 5-7). La notion de reste souligne le référent au tout duquel il a été retranché ou replacé.

7. En plus de la protection contre les personnes mal intentionnées que nous ne pouvons développer ici mais qui s’appuie sur ce mode de présence particulier.

\section{I'auteure}

Laure Carbonnel est docteure en anthropologie, associée au LESC (UMR 7186), post-doctorante au musée du quai Branly (2016-2017). Ses recherches portent sur les pratiques des kóródugaw au Mali et sur les processus de socialité engagés par la forme bouffonne.

\section{Iconographie}

Image d'ouverture. Saut d'un homme kóróduga chevauchant un cheval en bois. (C) Jethro Massey.

\section{Références}

Beaune, J.-C. 1999 «L'antidémiurgie: la matière vue du bas» in J.-C. Beaune dir. Le déchet, le rebut, le rien. Seyssel: Éditions Champ Vallon: 9-20.

Bloch, M. 2010 « Commensalité et empoisonnement » La pensée de midi 30: 81-89.

Carbonnel, L. 2015 Les kóródugaw du Mali. Comportements et groupements bouffons. Thèse de doctorat en anthropologie. Nanterre: Université Paris Ouest Nanterre la Défense.

Colleyn, J.-P. 2010 Les chevaux de la satire: les Korèdugaw du Mali. Montreuil : Éditions Gourcuff Gradenigo.

- 1988 Les chemins de Nya. Culte de possession au Mali. Paris: Éditions de l'EHESS.

— 1975 «Sur le chemin du village: l'initiation au Koro Minyanka », Journal de la Société des Africanistes 45 (1-2): 115-125.

Dumestre, G. 2011 Dictionnaire bambara-français suivi d'un Index abrégé français-bambara. Paris: Karthala.

Dumestre, G. \& T. Seydou 2007 Maléfices et manigances chroniques maliennes. Paris: Karthala.
1 à 9. (C) Laure Carbonnel \& Association des korèdugaw de Ségou.

10. C) Jethro Massey (jethromassey.com).

Duponchel, P. 1999 « Peinture à la terre, bògòlan du Mali », Journal des africanistes 69 (1) Des objets et leurs musées: 223-238.

Dupré, M.-Cl. 2007 «La réparation en Afrique: un moment dans la vie des objets » in G. Speranza dir. Objets blessés. La réparation en Afrique. Paris: Musée du quai Branly: 29-40

Gouhier, J. 1999 « La marge entre rejet et intégration » in J.-C. Beaune dir. Le déchet, le rebut, le rien. Seyssel: Éditions Champ Vallon: 80-89.

Jolly, E. 2004 Boire avec esprit. Bière de mil et société dogon. Nanterre: Société d'ethnologie.

Journet, O. 1981 «La quête de l'enfant. Représentation de la maternité et rituels de stérilité dans la société Diola de Basse-Casamance », Journal des africanistes 51 (1/2): 97-115

Malé, S. 2007 «Réparer le corps social » in Speranza G. dir. Objets blessés. La réparation en Afrique. Paris: Musée du quai Branly: $43-52$

Monteil, C. 1924 Les Bambara du Ségou et du Kaarta. Publications du Comité d'études historiques et 
scientifiques / Gouvernement général de l'Afrique occidentale française. Paris: Émile Larose Librairie-Éditeur.

Simmel, G. 1998 «Psychologie de la parure» in Id. La parure et autres essais. Paris: Éditions de la MSH: 79-88.
Zahan, D. 1960 Sociétés d'initiation Bambara. Le n'domo, le korè. Paris: La Haye, Mouton («Le monde d'Outre-mer passé et présent ». Première série. Études. 8).

Zonabend, F. 1999 «L'innomable et l'innomé » in J.-C. Beaune dir. Le déchet, le rebut, le rien. Seyssel: Éditions Champ Vallon: 90-98.

\section{Pour citer cet article}

Carbonnel, L. 2016 «Les rebuts captivants. Un nouvel ordre bouffon au cœur de la collectivité», TechniquesECulture 65-66 «Réparer le monde. Excès, reste et innovation», p. 370-385.

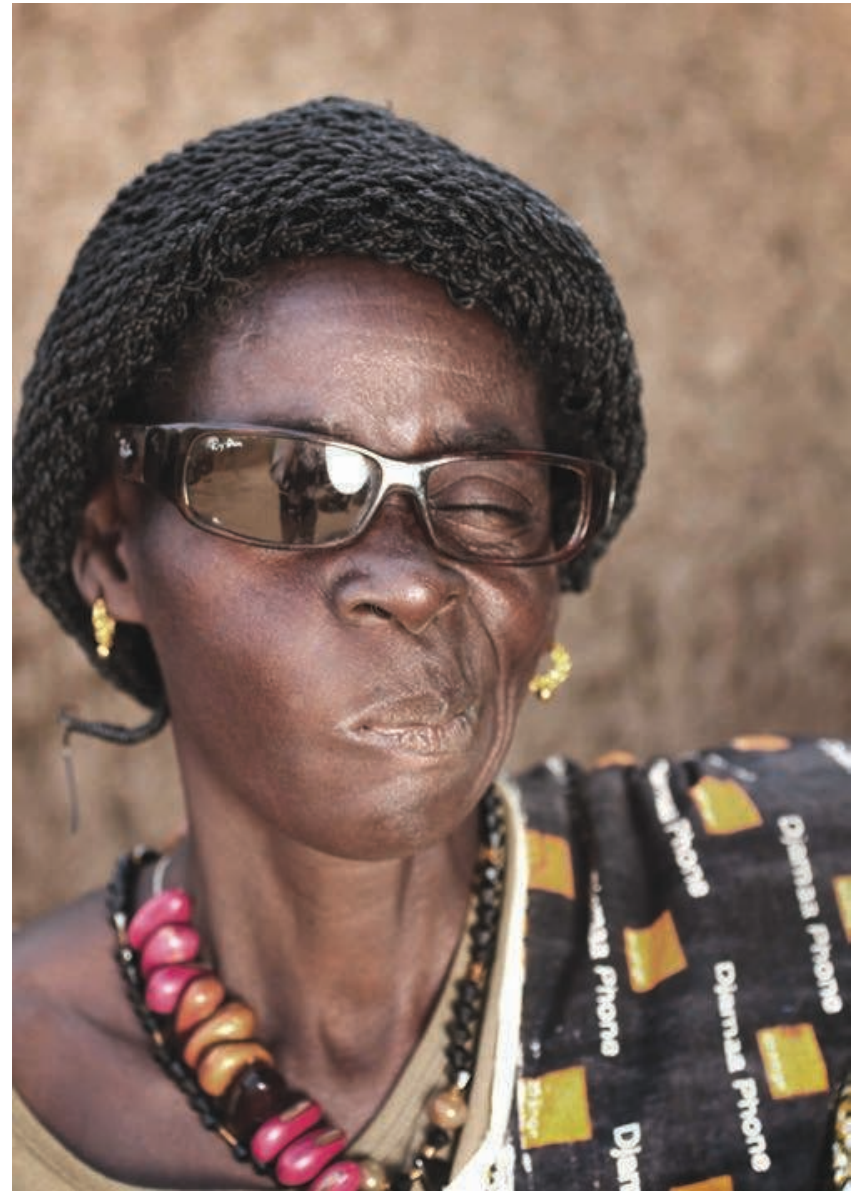

10. Le miroir bouffon mimiques et lunettes cassées. Ségou, 2011. 